\title{
Role of Prophylactic Phototherapy in the Management of very Low Birth weight Newborns with Hyperbilirubinemia in Comparison to Therapeutic Phototherapy with their Gender Oday Abbood Khalil (FIBMS) ${ }^{1}$, Hussam Mohamed Rafeeq (FIBMS) ${ }^{2}$ and Nadhim Ghazal Noaman $(\mathrm{PhD})^{3}$
}

\begin{abstract}
\end{abstract}
Background: Bilirubin associated brain damage is great in the preterm and low birth weight newborns. Phototherapy is able to control the level of bilirubin in low birth weight newborns. Objective:To show the role of prophylactic phototherapy in the management of very low birth weight newborns weighting (1-1.5) kg with hyperbilirubinemia in comparison to therapeutic method.

Patients and Methods: Across sectional study; Very low birth weight newborns weighting (1-1.5) kg were included in the study and admitted to neonatal care unit because of their very low birth weight and family consent had taken about admission and prophylactic phototherapy from some families so the total number of newborns was forty, twenty newborns were received phototherapy within nine hours of birth and lasted for seven days while twenty newborns were received phototherapy when indicated according to birth weight and continued to seventh day after birth, analysis was made for mean daily bilirubin level, duration of phototherapy, peak bilirubin level in the prophylactic and therapeutic groups and blood exchange need.

Results: The highest daily mean level of total serum bilirubin(TSB) of prophylactic group was $6.5 \pm 0.4 \mathrm{mg} / \mathrm{dl}$, which happened on the fifth day. In the therapeutic group, it was $8.7 \pm 0.5 \mathrm{mg} / \mathrm{dl}$ on the fifth day after birth. There was significant difference in the TSB values of prophylactic group which was less than that of therapeutic group from second to sixth days $(p<0.0001)$. The median duration of phototherapy in the treatment group was shorter than that of the prophylactic group.

Conclusion: Prophylactic phototherapy in very low birth weight preterm infants weighting (1-1.5) $\mathrm{kg}$ can significantly decrease jaundice after birth compared with the therapeutic group, also this study indicate that there is significant high TSB level among males in both groups in comparison to females.

Keywords:Prophylactic phototherapy, Very low birth weight newborns, Therapeutic phototherapy, Diyala.

Corresponding Author: drnadhimg@yahoo.com

Received: $17^{\text {th }}$ February 2019

Accepted: $25^{\text {th }}$ March 2019

\footnotetext{
1,2 Baquba Teaching Hospital - Diyala - Iraq.

${ }^{3}$ College of Medicine - University of Diyala -Diyala- Iraq.
} 


\section{Introduction}

Neonatal jaundice is common problem worldwide and it is effectively treated with phototherapy .Multiple phototherapy units are sometimes used to increase the light intensity and thus improve the efficacy of phototherapy [1].

Approximately $60 \%$ of term babies and $85 \%$ of preterm babies will develop clinically apparent jaundice [2,3].Most of these babies have so-called 'physiological jaundice', which typically becomes clinically apparent on day 3, peaks on day 5 to 7 and resolves by day 14. Physiological jaundice is usually benign, however if un conjugated serum bilirubin levels get too high, bilirubin can cross the blood brain barrier where it is neurotoxic, particularly to the auditory nerve and basal ganglia. Brain injury and long life disability can result. Because of this, it is important to identify those babies at risk of the rare complication of acute bilirubin encephalopathy and kernicterus $[2,3]$.

The premature newborn infant has an exaggerated form of physiologic jaundice with mean serum TSB concentrations reaching peaks of 10 to $12 \mathrm{mg} / \mathrm{dl}$ (171 to 205 $\mu \mathrm{mol} / \mathrm{L}$ ) or more with delay in reaching the maximum concentration as compared with full-term neonates (on the fifth and sixth day of life). Although this could be considered physiologic because of its occurrence in all preterm infants, the mean peak of un conjugated bilirubin concentrations higher than $10 \mathrm{mg} / \mathrm{dl}$ may be associated with acute bilirubin encephalopathy or kernicterus in certain high-risk, very low birth weight neonates[4,5].Thus, many neonatologists aggressively approach hyperbilirubinemia in very low birth weight (VLBW) neonate and use prophylactic or early phototherapy to prevent TSB levels from ever becoming sufficiently great to require exchange transfusion[6].

Risk factors for the development of sever hyperbilirubinemia and kernicterus include isoimmune hemolytic disease, glucose-6phosphate deficiency, asphyxia , significant lethargy, temperature instability, sepsis , acidosis, and hypoalbuminemia $(<3 \mathrm{~g} / \mathrm{dl})$ [7].

Phototherapy is the first line treatment for neonatal jaundice and is effective in most babies in reducing TSB level. Its efficacy depends on wavelength and luminance of the light source and the skin surface area illuminated by the light.[8] phototherapy has been effectively used as a relatively inexpensive and noninvasive method of treating neonatal hyperbilirubinemia[9,10].

It has been suggested that maintaining very low TSB levels with aggressive phototherapy might be associated with development of retinopathy of prematurity due to reducing antioxidant levels[11].Once the baby is receiving phototherapy, the suggested TSB level for ceasing would be a TSB level of $280 \mathrm{micro} \mathrm{mol} / \mathrm{L}$ for term babies, and for premature babies $50 \mathrm{micro} \mathrm{mol} / \mathrm{L}$ or more. [2,3].A rebound in total serum bilirubin levels can occur after phototherapy is discontinued[2].Babes born before 37 weeks gestation and those with haemolysis disease are at increased risk of clinically significant rebound. 
Role of Prophylactic Phototherapy in the Management of very Low Birth weight Newborns with

Hyperbilirubinemia in Comparison to Therapeutic Phototherapy with their Gender

Aim of the study was to show the role of prophylactic phototherapy in the management of very low birth weight newborns weighting(1-1.5)kg with hyperbilirubinemia in comparison to therapeutic methods and their gender effect.

\section{Patients and Methods}

This study was conducted in $\mathrm{Al}$-Batool Teaching Hospital in Diyala province-Iraq as cross sectional study ;in neonatal care unit from July to September in year 2018.

Forty neonates which had very low birth weight were included in this study. Special questionnaire was used included (name , gender, gestational age, weight, length , residency, type of feeding and TSB).

These neonates were of Iraqi race , healthy , breast and formula fed, delivered before 32th week of gestation, had birth weight ranging from $(1-1.5) \mathrm{kg}$ and following uneventful pregnancy.

Babes with hemolytic disease like ( $\mathrm{ABO}$ and $\mathrm{RH}$ incompatibility, positive Coombs test, G6PD deficiency), congenital and acquired infections, dehydration , Asphyxia , conjugated and persistence jaundice more than 14 days were excluded from the study . The enrolled neonates were divided in to two groups ;prophylactic photo therapy group (Initiate phototherapy during the first 9 hours and lasted for seven days after birth) and therapeutic group (started phototherapy when indicated according to birth weight and finished at seventh day after birth), analysis was made for mean daily bilirubin level, duration of phototherapy, peak bilirubin level in the prophylactic and therapeutic groups and blood exchange need.

\section{Statistical analysis}

Statistical analysis performed with the Analysis of data was carried out using the statistical package of SPSS-24 (Statistical Packages for Social Sciences- version 24). Data were presented in simple measures of frequency, percentage, mean standard deviation and range (minimum andmaximum values).

The significance of difference of different means (quantitative data) were tested using students-t-test for difference between two independent means or Paired sample. Statistical significance was considered whenever the $\mathrm{P}$ value was equal or less than 0.05 .

\section{Results}

Table(1)shows the demographic findings of both groups, in the prophylactic group, the number of newborns was twenty with mean birth weight $1.301 \pm 0.168(1.000-1.750) \mathrm{kg}$ while in the therapeutic group, the number of newborns was also twenty with mean birth weight $1.315 \pm 0.101 \mathrm{I}(1.150-1.500) \mathrm{kg}$, there were no significant difference with $p$ value(0.755).The mean length was $39.5 \pm 1.5(37-42) \mathrm{cm}$ in the prophylactic group and 40.2 $\pm 1.0(38-42)$ in the therapeutic group so there was no significant difference, $\mathrm{p}$. value(0.112). The head circumference was $28.0 \pm 1.0(26.5-29.5) \mathrm{cm}$ and 28.5 $\pm 0.9(26.5$ $30.0) \mathrm{cm}$ in the prophylactic and therapeutic groups respectively and the $\mathrm{p}$ value was 0.869 which showed no significant difference. 
The gestational age in the prophylactic and the therapeutic groups was 30 $\pm 1.2(28-32)$ and $30 \pm 1.1(27-32)$ weeks respectively with $\mathrm{p}$ value $(0.999)$ and there was no significant difference .So the prophylactic and the therapeutic groups were comparable regarding growth parameters and gestational age.
Table(2) showed that the fifth day after birth was the day of maximal mean TSB level in both groups, but the total mean TSB level in the prophylactic group was significantly lower on the second to sixth days after birth and significantly higher on the first and seventh days after birth than the therapeutic group.

Table (1): Demographic data of neonates in the prophylactic and therapeutic groups.

\begin{tabular}{||l||c||c||c||}
\hline Demographic data & $\begin{array}{c}\text { Prophylactic } \\
\text { N=20 }\end{array}$ & $\begin{array}{c}\text { Therapeutic } \\
\text { N=20 }\end{array}$ & P value \\
\hline \hline Birth weight(Kg) & $1.301 \pm 0.168$ & $1.315 \pm 0.101$ & $0.755(\mathrm{NS})$ \\
& $(1.000-1.750)$ & $(1.150-1.500)$ & \\
& & & \\
\hline Length(cm) & $39.5 \pm 1.5$ & $40.2 \pm 1.0$ & $0.112(\mathrm{NS})$ \\
& $(37-42)$ & $(38-42)$ & \\
\hline \hline Head circumference(cm) & $28.0 \pm 1.0$ & $28.5 \pm 0.9$ & $0.869(\mathrm{NS})$ \\
& $(26.5-29.5)$ & $(26.5-30.0)$ & \\
\hline \hline Gestation(weak) & $30 \pm 1.2$ & $30 \pm 1.1$ & $0.999(\mathrm{NS})$ \\
& $(28-32)$ & $(27-32)$ & \\
\hline *NS: no significant & & & \\
\end{tabular}

Table (2): Distribution of very low birth weight neonates with jaundice according to prophylactic and therapeutic treatment with their days of treatment .

\begin{tabular}{|c|c|c|c||}
\hline Age & $\begin{array}{c}\text { Prophylactic group } \\
\text { N=20 } \\
\text { Total serum bilirubin }\end{array}$ & $\begin{array}{c}\text { Therapeutic group } \\
\text { N=20 } \\
\text { Total serum bilirubin }\end{array}$ & P value \\
\hline \hline Day 1 & $4.5 \pm 0.4(4.0-5.6)$ & $4.1 \pm 0.3(3.5-4.6)$ & $0.002^{*}$ \\
\hline \hline Day 2 & $6.2 \pm 0.3(5.7-6.8)$ & $7.3 \pm 0.2(7.0-7.6)$ & $0.0001^{*}$ \\
\hline \hline Day 3 & $6.2 \pm 0.4(5.7-7.0)$ & $8.0 \pm 0.4(7.3-8.9)$ & $0.0001^{*}$ \\
\hline Day 4 & $6.5 \pm 0.2(6.1-6.9)$ & $8.5 \pm 0.4(8.0-9.0)$ & $0.0001^{*}$ \\
\hline \hline Day 5 & $6.5 \pm 0.4(6.0-7.7)$ & $8.7 \pm 0.5(7.9-9.9)$ & $0.0001^{*}$ \\
\hline \hline Day 6 & $6.4 \pm 0.2(6.1-6.8)$ & $7.2 \pm 0.3(6.7-7.7)$ & $0.0001^{*}$ \\
\hline \hline Day 7 & $6.2 \pm 0.2(5.8-6.6)$ & $6.0 \pm 0.3(5.5-6.4)$ & $0.015^{*}$ \\
\hline
\end{tabular}


Role of Prophylactic Phototherapy in the Management of very Low Birth weight Newborns with Hyperbilirubinemia in Comparison to Therapeutic Phototherapy with their Gender

TSB level of low birth weight neonates of therapeutic and prophylactic groups in comparison to the previous day showed that there were significant differences on second , fourth and seventh days among prophylactic group and significant difference on all days except fifth day among therapeutic group as show in Table(3) below.

Table (3): Distribution of very low birth weight neonates with jaundice according to the days of treatment with prophylactic and therapeutic groups in comparison to the previous day.

\begin{tabular}{|c||c||c||c|c||}
\hline Age & $\begin{array}{c}\text { Prophylactic group } \\
\mathbf{N = 2 0} \\
\text { Total serum bilirubin }\end{array}$ & P value & $\begin{array}{c}\text { Therapeutic group } \\
\mathbf{N = 2 0} \\
\text { Total serum bilirubin }\end{array}$ & P value \\
\hline Day 1 & $4.5 \pm 0.4$ & - & $4.1 \pm 0.3$ & - \\
\hline \hline Day 2 & $6.2 \pm 0.3$ & $0.0001^{*}$ & $7.3 \pm 0.2$ & $0.0001^{*}$ \\
\hline \hline Day 3 & $6.2 \pm 0.4$ & 0.867 & $8.0 \pm 0.4$ & $0.0001^{*}$ \\
\hline \hline Day 4 & $6.5 \pm 0.2$ & $0.002^{*}$ & $8.5 \pm 0.4$ & $0.0001^{*}$ \\
\hline \hline Day 5 & $6.5 \pm 0.4$ & 0.919 & $8.7 \pm 0.5$ & 0.085 \\
\hline Day 6 & $6.4 \pm 0.2$ & 0.342 & $7.2 \pm 0.3$ & $0.0001^{*}$ \\
\hline \hline Day 7 & $6.2 \pm 0.2$ & $0.002^{*}$ & $6.0 \pm 0.3$ & $0.0001^{*}$ \\
\hline
\end{tabular}

*significant difference two dependent means ( from previous day )using paired -t- test at 0.05 level.

Table(4)the demographic findings according to gender ,the number of males newborns of both prophylactic and therapeutic groups was ten while the females newborns number in both groups was ten also , there was no significant difference between gender regarding weight $(\mathrm{kg})$,length $(\mathrm{cm})$,head circumference $(\mathrm{cm})$ and gestational age(weeks) in the prophylactic group.In the therapeutic group ,there was significant difference in the length only (p. 0.023) which was more in females.

Also in the prophylactic males group ,there was significant difference with high mean total mean TSB level on all days except fifth day after birth while in the therapeutic group ,there was significant difference in all days except the first day after birth with high mean total serum bilirubin level among males as shown below. 
Role of Prophylactic Phototherapy in the Management of very Low Birth weight Newborns with Hyperbilirubinemia in Comparison to Therapeutic Phototherapy with their Gender

Oday Abbood Khalil

Table (4): The demographic findings and distribution of very low birth weight newborns with the days of treatment of prophylactic and therapeutic groups according to the gender.

\begin{tabular}{|c||c||c|c||c|c|c||}
\hline & \multicolumn{3}{|c||}{ Prophylaxis } & \multicolumn{3}{c||}{ Therapeutic } \\
\hline & Male & Female & P value & Male & Female & P value \\
\hline Weight $(\mathrm{Kg})$ & $1.262 \pm 0.133$ & $1.340 \pm 0.195$ & 0.310 & $1.289 \pm 0.102$ & $1.340 \pm 0.098$ & 0.274 \\
\hline Length $(\mathrm{cm})$ & $39.6 \pm 1.1$ & $39.5 \pm 1.8$ & 0.883 & $39.7 \pm 1.0$ & $40.6 \pm 0.8$ & $0.023^{*}$ \\
\hline \hline OFC $(\mathrm{cm})$ & $28.0 \pm 1.1$ & $28.2 \pm 0.9$ & 0.666 & $27.9 \pm 1.2$ & $28.2 \pm 0.5$ & 0.473 \\
\hline \hline GA $($ weeks) & $29.3 \pm 0.9$ & $29.8 \pm 1.4$ & 0.362 & $29.1 \pm 1.4$ & $30.0 \pm 0.7$ & 0.078 \\
\hline \hline TSB Day 1 & $4.8 \pm 0.4$ & $4.2 \pm 0.2$ & $0.001^{*}$ & $4.2 \pm 0.2$ & $4.0 \pm 0.3$ & 0.077 \\
\hline TSB Day 2 & $6.4 \pm 0.2$ & $6.0 \pm 0.2$ & $0.0001^{*}$ & $7.4 \pm 0.1$ & $7.2 \pm 0.2$ & $0.011^{*}$ \\
\hline TSB Day 3 & $6.4 \pm 0.4$ & $6.0 \pm 0.2$ & $0.005^{*}$ & $8.2 \pm 0.3$ & $7.9 \pm 0.3$ & $0.024^{*}$ \\
\hline \hline TSB Day 4 & $6.6 \pm 0.2$ & $6.4 \pm 0.2$ & $0.025^{*}$ & $8.8 \pm 0.1$ & $8.2 \pm 0.2$ & $0.0001^{*}$ \\
\hline TSB Day 5 & $6.6 \pm 0.5$ & $6.4 \pm 0.4$ & 0.328 & $8.9 \pm 0.6$ & $8.4 \pm 0.3$ & $0.018^{*}$ \\
\hline \hline TSB Day 6 & $6.5 \pm 0.2$ & $6.3 \pm 0.1$ & $0.015^{*}$ & $7.4 \pm 0.2$ & $7.0 \pm 0.2$ & $0.0001^{*}$ \\
\hline TSB Day 7 & $6.3 \pm 0.2$ & $6.1 \pm 0.2$ & $0.046^{*}$ & $6.2 \pm 0.1$ & $5.8 \pm 0.2$ & $0.0001^{*}$ \\
\hline
\end{tabular}

*Significant difference between two independent means (males and females) using students-t-test at 0.05 level.

\section{Discussion}

This study showed that the mean total serum bilirubin level was significantly high in the therapeutic group in compared to the prophylactic group on second to sixth days of life and significantly low on first and last days which showed the effect of prophylactic phototherapy to limit the significant increase in TSB levels especially if other risk factors may coexist or appeared later like sepsis and prevent the reach to the critical level and avoidance the need for exchange transfusion while the level of TSB is higher in the first day among prophylactic group due to the selection of very low birth weight newborns with significant high TSB level and put them on prophylactic phototherapy, in spite of this point, the prophylactic phototherapy played a role to significant decrease TSB level in the later days in comparison to the therapeutic method. The peak TSB level of both groups occurred on fifth day after birth but the level of TSB had significant difference ( $p$ 0.0001). In a study done by Remin 1 study[14] in Iran showed significant difference with high TSB level in the treatment group which go with study, the peak mean TSB level in Remin study[14] in the prophylactic group was on the third day and in the treatment group was on the fourth day, the results of present study were nearly consistent with study in Iran , this may be due to similar range of birth weight $(1-1.5) \mathrm{kg}$ and could be due to similar time of starting and ending of phototherapy in both groups. In another study done in brazil by Leite [12], it showed that the peak mean TSB level in the early phototherapy group was on the seventh day and in the late phototherapy group was on the second day and had significant difference $(\mathrm{p}<0.01)$, this was different from present study and this may be due to difference in the weight range of neonates and the duration of phototherapy 
, so the results of this study showed that the prophylactic phototherapy could prevent the significant increase of the TSB level in the following days .

Curtis Cohen et al study[6] showed no significant difference in the peak mean TSB levels in group who received phototherapy after birth and group who received phototherapy if TSB reached $5 \mathrm{mg} / \mathrm{dl}$.this may be due to early time of starting phototherapy in the treatment group $(5 \mathrm{mg} / \mathrm{dl})$ which was close to prophylactic group TSB level.

In a study conducted by Tripathy et al.[13], there was no significant difference in the age of reaching peak TSB levels in control and prophylactic phototherapy groups indicating that prophylactic phototherapy is not beneficial in preterm low birth neonates, this results differed from that of other studies, this may be due to different environments, study designs, weight range and the time of starting phototherapy in the therapeutic group.

This study also proved that there was significant high TSB level among males in the prophylactic group in all days except fifth day and significant high TSB level in males in the therapeutic group in all days and this results were agree with study done by TIOSECO JA et al[ 15 ].

\section{Conclusions}

In conclusion, prophylactic phototherapy in very low birth weight preterm infants who weigh between (1-1.5) $\mathrm{kg}$ can significantly decrease TSB level after birth compared with the therapeutic group especially when other causes of high TSB (which had been excluded from this study) may coexist and complicate the condition, so the prophylactic phototherapy is beneficial to decrease TSB level. Also, This study indicate that there is significant high TSB level among males in both groups in comparison to females which mean that the gender play a role in TSB level in very low birth weight neonates.

\section{Recommendation}

This study recommend the use of prophylactic phototherapy in very low birth weight newborns weighting from (1-1.5) kg.

\section{References}

[1]Sarici SU , Alpay F , Unay B . Double versus single phototherapy in term newborns with significant hyperbilirubinemia J Trop pediatr $2000 ; 46: 36-9$.

[2]Subcommittee on hyperbilirubinemia, American Academy of Pediatrics. Management of hyperbilirubinemia in the newborn infant 35 or more weeks of gestation, Clinical Practice Guideline. Pediatrics 2004, 114(1): 297-316.

[3]NHS National Institute for Health and Clinical Excellence. Neonatal Jaundice:AclinicalGuideline.<http://guidance .nice.org.uk/CG98/Guidance/pdf/English.

[4]Maisels MJ. What's in a name? Physiologic and pathologic jaundice: the conundrum of defining normal bilirubin levels in the newborn. Pediatrics 2006; 118(2):805-7.

[5]Watchko JF, Maisels MJ. Jaundice in low birth weight infants: pathobiology and outcome. Arch Dis Child Fetal Neonatal Ed 2003;88(6): F455-8. 
[6]Curtis-Cohen M, Stahl GE, Costarino AT. Randomized trial of prophylactic phototherapy in infants with very low birth weight. J Pediatr 1985;107(1):121-4.

[7]American Academy of pediatrics. Management of hyperbilirubinemia in the Newborn infants 35 or more weeks of Gestation .Pediatrics 2004.114(1):297-316. [8]Maisels MJ, McDonagh AF. Phototherapy for Neonatal Jaundice. N Engl J Med 2008; 358(9): 920-928.

[9] Slusher TM, Olusanya BO, Vreman HJ , Brearley AM, Vaucher YE, Lund TC, et al. A Randomized Trial of phototherapy with filtered Sunlight in Africa Neonates. N Engl J Med. 2015 sep 17.373(12):1115-24.

[10] Slusher TM , Vreman HJ , Olusanya BO , Wong RJ , Brearley AM , Vaucher YE, et al. Safety and efficacy of filtered sunlight in treatment of jaundice in Africa neonates. Pediatrics. 2014 Jun . 133(6):e 1568-74.

[11]Maisels MJ, Watchko JF. Treatment of jaundice in low birth weight infants. Arch Dis Child Fetal Neonatal Ed 2003;88(6):F459-63.

[12]Leite Md, Facchini Fp. Evaluation of two guidelines for the management of hyperbilirubinemia in newborn babies weighing less than 2,000 g. J Pediatr (Rio J) 2004;80(4): 285-90.

[13]Tripathy S, Saili A. Effect of prophylactic phototherapy on neonatal hyperbilirubinemia of prematures . Indian J Med Sci 2006;60(9):385-7. [14]Remin I, Majid M, Seyedeh- Sarah N. Comparison of two phototherapy methods prophylactic VS therapeutic in management of hyperbilirubinemia in very low birth weight newborns. Iran J Pediatric Dec 2011; 21 ( 4 ): 425-430. [15]Tioseco JA , Aly H , Milner J , Patel K , El-Mohandes AA.Dose gender affect neonatal hyperbilirubinemia in low birth weight infants.Pediatr Crit Care med.2005 Mar;6(2):171-4. 